\title{
The Scale-Relativity Program*
}

\author{
L. Nottale \\ CNRS, DAEC, Observatoire de Paris-Meudon, \\ F-92195 Meudon Cedex, France
}

November 21, 2002

\begin{abstract}
The theory of scale relativity is an attempt at developing the consequences of giving up the implicit hypothesis of space-time differentiability. One of these consequences is that space-time becomes fractal, i.e., explicitly resolution-dependent. The principle of relativity is extended in order to be also valid for scale transformations of space-time resolutions. In the present contribution, we mainly focus on the general structure of the theory and on its possible future goals. This description is completed by a development of three specific points: (i) a new demonstration of the Schrödinger equation in which all terms are kept; (ii) a method of entropy maximization allowing one to unveil the fractal geometry from the quantum behavior; (ii) a discussion of the metric forms in scale-relativity. (C)1999 Elsevier Science Ltd. All rights reserved
\end{abstract}

\section{INTRODUCTION}

The present paper is intended to provide the reader with a general view of the general project that we have called "scale-relativity theory". We shall try to give a hint about the overall structure of the theory, in order to make apparent what remains to be done. Some conjecture about its future ways of development will be given, and also some comments about specific points which have not been developed up to now. A more detailed account of the present status of the theory and of its results already obtained can be found in the book [1], and in the more recent review paper [2].

The theory of scale relativity is founded on an attempt at giving up the hypothesis of differentiability of space-time coordinates. One of the first consequence of such an opening of the possibilities is a generalization of the geometric structure of space-time from Riemannian to fractal [3], [4]. The present theory

${ }^{*}$ Published in Chaos, Solitons 85 Fractals, Vol. 10, No. 2-3, pp. 459-468 (1999) 
of generalized relativity assumes class $C 2$ coordinates, i.e., it assumes that one can define velocities and accelerations [5]. In our attempt of generalization, we assume only class $C 0$ coordinates, i.e., that space-time is a pure continuum, which is not a priori differentiable. El Naschie [6] tries to go even further, and gives up the continuity hypothesis in his concept of Cantorian fractal space-time.

\section{ON RELATIVITY THEORIES}

Let us first recall what is, in our opinion, the essence of relativity theories. It can be expressed in Galileo's discovery that "motion is as nothing". Motion does not exist by itself, but only as a relative property that characterizes a couple of bodies. It has no meaning for a single object. More recently, Einstein reached the same kind of conclusion concerning gravitation. Gravitation does not exist by itself, but only as a consequence of the choice of the reference system. In the free-fall system, gravitation locally disappears, leaving only inertial motion, i.e. the laws of motion for a free body unaffected by a force or a field.

This essence of relativity is particularly evidenced in the relations between the Einstein's three founding principles for the theory of general relativity, namely, the principle of relativity, the principle of covariance and the principle of equivalence.

The principle of relativity is the statement that the laws of nature be valid in any reference system, whatever its state [5].

The principle of covariance is its mathematical translation: it requires that the equations of physics keep the same, simplest possible form, in coordinate transformations. Note that one can distinguish between a weak covariance principle, according to which the form of the equations must only be the same in a new cordinate transformation as it was in simpler ones; and a strong covariance principle, that pushes to its end the argument, requiring that the equations of physics should take the simplest of all its form, i.e. that of free, inertial motion.

The principle of equivalence, when adapted to gravitation, states that a gravitational field is locally equivalent to an accelerated motion. Its consequence is the possibility to absorb the gravitational field in the choice of the reference system [7]. But one may try to generalize it, by requiring that, whatever the physical object or field considered, one can always find a state of the reference system in which it could be suppressed.

In this regard, when seen either from the strong principle of covariance, or from this generalized principle of equivalence-absorption, the ultimate goal of the theories of relativity would be to reach a description in which the various physical objects would not exist by themselves, but would see their very existence come from changes of the reference system. In the proper system, the description would be that of pure free motion in vacuum.

It is clear that today's physics is still very far from approaching such a goal (that could well be an horizon for physics). The theory of scale relativity is an 
attempt at making some small steps in this direction, concerning in particular the nature of the quantum effects.

\section{STRUCTURE OF THE THEORY}

\subsection{General structure}

The theory of scale relativity is constructed by completing the standard laws of classical physics (motion in space / displacement in space-time) by new scale laws (in which the space-time resolutions are used as intrinsic variables, playing for scale transformations the same role as played by velocities for motion transformations). We hope such a stage of the theory to be only provisional, and the motion and scale laws to be treated on the same footing in the final theory (see Castro [8]). However, before reaching such a goal, one must realize that the various possible combinations of scale laws and motion laws lead to a large number of sub-sets of the theory to be developed. Indeed, three domains of the theory are first to be considered:

(i) pure scale-laws: description of the internal structures of a non-differential space-time at a given point / event;

(ii) induced effects of scale laws on the equations of motion: generation of the quantum mechanics as mechanics on a nondifferentiable space-time;

(iii) scale-motion coupling: effects of dilations induced by displacements, that we tentatively interpret as gauge fields (only the case of the electromagnetic field has been considered up to now) [9], [2].

Several levels of the description of scale laws (point i) can be considered. These levels are quite parallel to that of the historical development of the theory of motion:

(i1) Galilean scale-relativity: standard laws of dilation, that have the structure of a Galileo group (fractal power law with constant fractal dimension). When the fractal dimension of trajectories is $D=2$, the induced motion laws are that of standard quantum mechanics $[1],[10],[2],[11]$.

(i2) Special scale-relativity: generalization of the laws of dilation to a Lorentzian form [12]. The fractal dimension becomes a variable, and plays the role of a fifth dimension. An impassable length-time scale, invariant under dilations, appears in the theory; it replaces the zero, owns all its physical properties (an infinite energy-momentum would be needed to reach it), and plays for scale laws the same role as played by the velocity of light for motion. 
(i3) Scale-dynamics: while the first two cases correspond to "scale freedom", one can also consider distorsion from strict self-similary that would come from the effect of a "scale-force" or "scale-field" [13], [14].

(i4) General scale-relativity: in analogy with the field of gravitation being ultimately attributed to the geometry of space-time, a future more profound description of the scale-field could be done in terms of Riemann geometry of the fifth-dimensional scale space.

(i5) Quantum scale-relativity: the above cases assume differentiability of the scale transformations. If one assumes them to be continuous but, as we have assumed for space-time, non-differentiable, one is confronted for scale laws to the same conditions that lead to quantum mechanics in space-time. One may therefore conjecture that quantum mechanical scale laws could be constructed in a future work.

The possible complication of the theory becomes apparent when one realizes that these various levels of the description of scale laws will lead to different levels of induced dynamics (point ii) and scale-motion coupling (iii), and that other sublevels are to be considered, depending on the status of motion laws (non-relativistic, special-relativistic, general-relativistic).

\subsection{Domains of application of the theory}

A new complication comes from the fact that three domains of application of the theory can be considered:

(a) Microphysics: new scale-relativistic effects are expected in the realm of elementary particle physics at high energy $(\Delta x \rightarrow 0, \Delta t \rightarrow 0)$. We expect $\hbar$ to have an effective value that varies with scale beyond the top quark energy [2]. Moreover, new experiments can also be considered: in analogy with the fact that the free fall reference system for gravitation is an accelerating system, one may conjecture that some particle-fields could be absorbed in coordinate systems characterized by a "scale-acceleration" [13], [14]. Recall indeed that a resolution is identified, in the scale-relativity theory, with a scale-velocity, which is a derived quantity; one can go one step further with the concept of scale-field and introduce a second-derived quantity, the scale-acceleration. In such a new experiment, the resolution of the measurement apparatus should be variable in space-time, while the scale dependance should be no longer self-similar.

(b) Cosmology: another natural domain of application of scale-relativity is very large scales. We have proposed that Lorentzian scale laws could also be valid toward the large scales, implying the existence of a finite, maximal resolution, 
impassable, invariant under dilations, owning the physical properties of the infinite, which we have identified with the scale of the cosmological constant [1] [15]. In this new framework, new solutions can be brought to the problems of the vacuum energy density, of Mach principle and of the large number hypothesis [2].

(c) Complex systems: we have suggested ([1] Chapt.7, [14]) that a general theory of structure formation should take a quantum mechanical-like form. Indeed, several structures, in particular in the biological domain, are density waves rather than solid objects. For describing such a system, one is no longer interested in the individual trajectories, but instead in the overall structure, that the individual particles only cross. Our proposal is to go even further and, basing ourselves on the fractal character of individual trajectories and on the fundamental irreversibility of such a process at the infinitesimal level, to describe complex structures as density waves in term of probability amplitudes, themselves being solutions of a generalized Schrödinger equation [14]. An application of this approach to the problem of the formation and evolution of gravitational structures has given several results in the recent years, at scales ranging from planetary systems to large scale structures of the universe: solar system [1] Chapt.7.2, [16], extra-solar planetary systems [17], planets around pulsars [18], obliquities and inclinations in the solar system [19], satellites of giant planets [20] [21], binary stars [22], binary galaxies [23], large scale distribution of galaxies [2].

Let us finally remark that large parts of the theory (i2, iii) are still uncomplete, since they are up to now expressed only in the framework of a reduced number of dimensions. Only a very small fraction of the theory has been developed up to now, and far more remains to be done, in particular concerning the levels (ii) $+(\mathrm{i} 2-\mathrm{i} 5)$ and (iii).

\subsection{Induced dynamics}

Let us be more specific about the structure of the sub-theory that concerns the dynamics induced by scale laws (ii). We have found that the underlying structures of space-time transform the classical Hamilton-Jacobi equation into a Schrödinger equation [1]. The case of the Klein-Gordon equation has been studied in [9], [2], [24], and the Dirac equation by Ord [3] in his spiral model and by Pissondes [24]. The giving up of the hypothesis of differentiability of space-time coordinates leads to three effects (at least):

(1) The number of geodesics become infinite. This result leads one to jump to a statistical, fluid-like description.

(2) Each geodesic becomes a fractal curve. As a consequence, new terms are to be added in the differential equations of motion.

(3) The symmetry $d t \rightarrow-d t$, which was a hidden symmetry of the standard theory, is broken by non-differentiability. This leads to a two-valuedness of the average velocity vector, which is, in our framework, the origin of the complex 
nature of the probability amplitude.

Even concerning this reduced problem, the theory remains highly uncomplete. Much remains to be done. The violation of the time symmetry at the infinitesimal level may be completed by a violation of parity, leading to additional multiple-valuedness of the basic variables. Among other possibilities of future developments, one may also wonder whether, while a two-valuedness of the velocity is implied by non-differentiability, a three-valuedness of the acceleration would be needed.

\section{NEW DEMONSTRATION OF SCHRODINGER EQUATION}

In previous works, we have obtained the Schrödinger equation by taking average quantities. Let us briefly show here how one can make a complete calculation where all terms are kept.

One starts with elementary displacements $d X$ that are described in terms of an average $d x$ and a fluctuation $d \xi$ :

$$
d X=d x+d \xi .
$$

While $d x=v d t$, the $d \xi$ are fractal fluctuations of zero mean which can be written:

$$
d \xi=a \sqrt{2 \mathcal{D}}\left(d t^{2}\right)^{\frac{1}{2 D}}+K\left(d t^{2}\right)^{\frac{k}{2}},
$$

where $D$ is the fractal dimension of the trajectory, $\langle a\rangle=0,\left\langle a^{2}\right\rangle=1$, $<K>=0$ and $k>1$. Recall that one of the basic methods of scale-relativity consists of treating the differential elements as full variables. Non-standard analysis is one possible mathematical framework allowing one to develop such a method in a rigorous way [25].

It is remarkable that the $d \xi$ 's exist only at the differential level, but do not contribute to the macroscopic space-time coordinates, since they are of zero mean. Hence, at the level of the space-time coordinates, $X$ and $x$ are indistinguishable, while at the level of their derivative the $d X$ 's no longer reduce to the classical differential $d x$ 's. The new variables $d \xi$ are those that carry the underlying information about the fractal inner structure of space-time and are coupled to the fractal dimension. The scale laws to be constructed are those that describe their behavior. Strictly, they go beyond space-time. The classical macroscopic space-time $(x, y, z ; t)$ emerges from the far more complicated structure $\left(d x, d y, d z ; d t:: d \xi_{x}, d \xi_{y}, d \xi_{z}, d \xi_{t} ; \delta\right)$. Note also that the above quantity $\mathcal{D}$ which, in the simple case leading to standard quantum mechanics is a simple parameter (it is related to the Compton length: $\mathcal{D}=\hbar / 2 m$ ), is expected to become a full field in the general case, in connection with a variable fractal dimension. 
Let us go on with this 'exact' calculation. Consider a function $f(X(t), t)$, and expand it in terms of Taylor expansion:

$$
\frac{d f}{d t}=\frac{\partial f}{\partial t}+\frac{\partial f}{\partial X} \frac{d X}{d t}+\frac{1}{2} \frac{\partial^{2} f}{\partial X^{2}} \frac{d X^{2}}{d t}+\frac{1}{6} \frac{\partial^{3} f}{\partial X^{3}} \frac{d X^{3}}{d t}+\ldots
$$

The velocity is given by:

$$
\frac{d X}{d t}=v+a \sqrt{2 \mathcal{D}}\left(d t^{2}\right)^{\frac{1}{2 D}-\frac{1}{2}}+K\left(d t^{2}\right)^{\frac{k-1}{2}} .
$$

This means that it contains a divergent term of zero-mean, $w=a \sqrt{2 \mathcal{D}}\left(d t^{2}\right)^{\frac{1}{2 D}-\frac{1}{2}}$ which manifests the non-differentiability. Concerning the fluctuations, one obtains:

$$
\frac{d X^{2}}{d t}=2 a^{2} \mathcal{D}\left(d t^{2}\right)^{\frac{1}{D}-\frac{1}{2}}+2 a \sqrt{2 \mathcal{D}} v\left(d t^{2}\right)^{\frac{1}{2 D}}+v^{2} d t+2 v K\left(d t^{2}\right)^{\frac{k}{2}}+K^{2}\left(d t^{2}\right)^{k-\frac{1}{2}}+\ldots
$$

$$
\frac{d X^{3}}{d t}=a^{3}(2 \mathcal{D})^{3 / 2}\left(d t^{2}\right)^{\frac{3}{2 D}-\frac{1}{2}}+\ldots
$$

We verify that a finite contribution remains up to terms in $d X^{D} / d t$, while the preceeding ones contain infinite contributions. In what follows, we shall only consider the case when the fractal dimension $D$ is close to 2 . The derivative of a function $f$ can now be written:

$$
\frac{d f}{d t}=\frac{\partial f}{\partial t}+(v+w) \frac{\partial f}{\partial x}+\mathcal{D} \frac{\partial^{2} f}{\partial x^{2}}\left(d t^{2}\right)^{\frac{1}{D}-\frac{1}{2}}+K^{\prime}\left(d t^{2}\right)^{\frac{k^{\prime}}{2}}+\ldots
$$

with $k^{\prime}>0$. Let us now introduce the two-valuedness of the derivative, that comes from the fundamental irreversibility induced by the giving up of differentiability [14]. The two velocities $v$ and $w$ are actually each of them two-valued. This comes from the breaking of the infinitesimal time symmetry $d t \leftrightarrow-d t$. We therefore introduce complex velocities:

$$
\mathcal{V}+\mathcal{W}=\left(\frac{v_{+}+v_{-}}{2}-i \frac{v_{+}-v_{-}}{2}\right)+\left(\frac{w_{+}+w_{-}}{2}-i \frac{w_{+}-w_{-}}{2}\right)
$$

The new divergent complex velocity is of zero mean $\langle\mathcal{W}\rangle=0$. One can verify that its square is also of zero mean: $\left\langle\mathcal{W}^{2}\right\rangle=0$. This is an important result for the equation of motion. Indeed, the complete Lagrange function for a free particle is written $\mathcal{L}=\frac{1}{2} m(\mathcal{V}+\mathcal{W})^{2}$, and it reduces in the mean to the form that we have used in our previous demonstrations, $\mathcal{L}=\frac{1}{2} m \mathcal{V}^{2}$ [1]. An additional term must be taken into account in the 'quantum-covariant' derivative, which now writes for $D=2$ :

$$
\frac{\bar{d}}{d t}=\frac{d}{d t}+\mathcal{W} \cdot \nabla=\frac{\partial}{\partial t}+(\mathcal{V}+\mathcal{W}) \cdot \nabla-i \mathcal{D} \Delta
$$


plus terms that vanish when $d t \rightarrow 0$. The equation of motion of a free "particle" (i.e., in our framework, the second order differential geodesic equation) therefore writes:

$$
\frac{\bar{d}(\mathcal{V}+\mathcal{W})}{d t}=0
$$

New divergent terms of zero mean have been added to our previous equation $d \mathcal{V} / d t=0$ :

$$
\frac{d \mathcal{V}}{d t}+\left(\frac{\partial \mathcal{W}}{\partial t}+\nabla(\mathcal{V} \cdot \mathcal{W})+\mathcal{W} \cdot \nabla \mathcal{W}-i \mathcal{D} \Delta \mathcal{W}\right)=0
$$

This equation can be written under the form:

$$
\frac{d \mathcal{V}}{d t}+F \sqrt{2 \mathcal{D}} d t^{-\frac{1}{2}}=0
$$

where $\langle F\rangle=0$. It becomes now clear, thanks to our keeping of all the non vanishing terms, even those of zero mean, that the averaging that leads to the Schrödinger equation is made on the set of individual geodesics. After some calculations and assuming that $F=\nabla G$, with $\langle G\rangle=0$, we find that this equation can be integrated in the form of a generalized Schrödinger equation (see [1] [2]:

$$
\mathcal{D}^{2} \Delta \psi+i \mathcal{D} \frac{\partial \psi}{\partial t}+G d t^{-\frac{1}{2}} \psi=0
$$

Taking the average yields the free particle Schrödinger equation. An interesting consequence of this result is also that one could introduce fluctuations such that $<G>=\phi d t^{1 / 2}$, which would be classically indistinguishable from $<G>=0$ but would yield a potential term in the Schrödinger equation, whose origin would be purely geometric (see Ord [26] for an equivalent result using a random walk model).

\section{GENERAL LINK BETWEEN HEISENBERG- DE BROGLIE RELATION AND FRACTAL BEHAVIOR}

Finkel [27] has suggested to use the information entropy for constructing generalized Heisenberg inequalities. His method consists of building the extremum state that achieves the wanted minimum. It can be used to give a general solution to the problem, which dates back to the Abbott and Wise work [28], of translating quantum mechanical relations in terms of fractal geometric behavior. As emphasized in [1], the key to this problem is to understand the relation, for a given variable $X$, between $\langle|X|\rangle^{2},\langle X\rangle^{2}$ and $\left\langle X^{2}\right\rangle$. This question 
remains essential even nowadays: for example, in his proposal for an experiment to measure the Hausdorff dimension of quantum mechanical trajectories, Kröger [29] assumes that:

$$
<|\Delta x|>^{2}=<(\Delta x)^{2}>
$$

As already shown in [1], this relation is not strictly valid, but it is an extremum relation. Let us establish again this result using Finkel's method. Such a relation is important to be known, since it yields not only the small scale fractal behavior and the identification of the fractal dimension $D=2$, but also the value of the transition length toward non fractal behavior at large scale (i.e., the quantumclassical transition).

Consider two observables $A$ and $B$ and their averages, $\langle A\rangle=\sum_{a} \omega_{a} a$ and $<B>=\sum_{b} \omega_{b} b$. For example, for obtaining the standard position-momentum Heisenberg relation, one will take $A=(x-<x>)^{2}$ and $B=(p-<p>)^{2}$.

The information entropy is given by

$$
S(\varphi)=-\sum_{a} \omega_{a} \ln \omega_{a}
$$

with

$$
\omega_{a}=|<a| \varphi>\left.\right|^{2} ;<\varphi \mid \varphi>=1
$$

We shall now require $\langle A>$ to be extremum:

$$
\delta<A>=0
$$

Finally, Finkel's method amounts to maximize the entropy (15) subject to the constraints (16) and (17). The solution of such an optimization is:

$$
<a \mid \varphi>=\exp \left(\frac{\Omega-\lambda a}{2}\right) ; \Omega=-\ln \left(\sum_{a} e^{-\lambda a}\right) ;<A>_{e x}=\frac{\partial \Omega}{\partial \lambda} .
$$

For example, when it is applied to $\langle A\rangle=\delta x$ and $\langle B\rangle=\delta p$, this method yields the position-momentum uncertainty relation for intervals:

$$
\delta x \delta p \geq \frac{\hbar}{\pi}
$$

This minimum is achieved by the ground state of the harmonic oscillator.

Let us now apply this method to typical quantum paths in the non-relativistic case. The length measured along a typical (nondeterministic) quantum trajectory is

$$
\mathcal{L} \propto<|v|>\propto<|p|>
$$


in the non-relativistic case. The problem posed here is therefore finding a Heisenberg relation for the absolute value of a variable, $A=|z|$, rather than for the variable $z$ itself. The extremum is given by:

$$
<a \mid \varphi>=\exp \left(\frac{\Omega-\lambda|z|}{2}\right)
$$

with

$$
\Omega=-\ln \left(\int e^{-\lambda|z|} d|z|\right)=\ln \left(\frac{\lambda}{2}\right) .
$$

The maximized mean value is therefore:

$$
<A>_{e x}=\frac{\partial \Omega}{\partial \lambda}=\frac{1}{\lambda}
$$

The probability distribution that achieves the extremum is:

$$
\omega_{e x}(|z|)=e^{\Omega-\lambda|z|}=\frac{1}{2<|z|>} e^{-|z| /<|z|>}
$$

Therefore we finally find the extremal relation:

$$
<|z|>^{2}=<z^{2}>
$$

When this method is applied to $z=\Delta x$, it yields the inequality:

$$
<|\Delta x|>^{2} \leq<\Delta x^{2}>
$$

Let us now consider again the problem of typical quantum paths. Choosing $z=p$ and knowing that $<(\Delta p)^{2}>=<p^{2}>-<p>^{2}$, we obtain the extremum relation:

$$
<|p|>^{2}=<p>^{2}+<(\Delta p)^{2}>
$$

Now $\left\langle p>\right.$ yields the de Broglie length, $\lambda=\hbar /\left\langle p>\right.$, while $\left\langle(\Delta p)^{2}\right\rangle$ is linked through the Heisenberg relation to the position dispersion or resolution, $\delta x \approx \hbar / \Delta p$. Since the length during a total time interval $T$ is $\mathcal{L}=<|p|>T / m$, we obtain:

$$
\mathcal{L}=<v>T\left[1+\left(\frac{\lambda_{x}}{\Delta x}\right)^{2}\right]^{\frac{1}{2}}
$$

This is easily generalized to 3 coordinates:

$$
V=<v>\left(1+\frac{\lambda^{2}}{\sigma^{2}}\right)^{\frac{1}{2}}
$$

with $\lambda^{-2}=\lambda_{x}{ }^{-2}+\lambda_{y}{ }^{-2}+\lambda_{z}{ }^{-2}$ and $\sigma^{-2}=\sigma_{x}{ }^{-2}+\sigma_{y}{ }^{-2}+\sigma_{z}{ }^{-2}$. 
This result can now be compared with the prediction from a fractal model of trajectory. The length of a fractal trajectory is given by:

$$
\left.\mathcal{L}=\int\left[1+\left(\frac{d X}{d t}\right)^{2}\right)\right]^{\frac{1}{2}} d t
$$

Thanks to the general relation between time resolution and length resolution on a fractal curve, $\delta X \approx \lambda^{D-1} \delta t$, we can integrate Eq. 30, and we obtain the final scale dependence:

$$
\mathcal{L} \approx<v>T\left[1+\left(\frac{\lambda}{\delta X}\right)^{2(D-1)}\right]^{\frac{1}{2}}
$$

We recognize here the quantum result, which allows us to identify the transition scale $\lambda$ with the de Broglie length and to fix the fractal dimension to $D=2$, in accordance with the Abbott-Wise and subsequent results (see e.g. [1]).

\section{METRICS IN SCALE RELATIVITY}

It seems clear that, in the strict usual meaning, there can be no metric in scale relativity, since the space-time coordinates are no longer differentiable. However, this problem is shunted by the introduction of resolutions as explicit variables. As a consequence, the metric is not unique as in general relativity, but, instead, several metric forms can be introduced. One metric is that of the standard space-time coordinates, $(x, y, z ; t)$. A second one, the scale metric, concerns the $d \xi$ 's and the fractal dimension. A third one is the metric induced in space-time by scale laws. Let us briefly comment about these last two metrics.

\subsection{Pissondes's metric form}

The problem of the metric is fundamental in relativity theories since it amount to find the invariant. The scale-relativistic approach to the problem of a spin zero particle in relativistic quantum mechanics (Klein-Gordon equation) has been first considered in ref. [9] (see also [2]), then it has been improved by Pissondes [24]. The free particle Klein-Gordon equation can be obtained from a geodesics equation:

$$
\frac{d \mathcal{V}_{j}}{d s}=0
$$

where the covariant derivative is written:

$$
\frac{d}{d s}=\left(\mathcal{V}^{k}+\frac{1}{2} i \lambda \partial^{k}\right) \partial_{k}
$$

In [9], we had the hope that the standard form of the metric in special and general relativity, namely $v_{k} v^{k}=1$, could be conserved in terms of our new 
complex velocity $\mathcal{V}$. However, it has been shown by Pissondes [24] that the equation $\mathcal{V}_{k} \mathcal{V}^{k}=1$ is wrong in scale relativity, and that the metric form for a spin zero particle instead writes :

$$
\mathcal{V}_{k} \mathcal{V}^{k}+i \lambda \partial_{k} \mathcal{V}^{k}=1
$$

where $\lambda=\hbar / m$ characterizes the Compton scale. It may be interesting to compare this Pissondes metric form to a scale invariant Finsler metric form (that yields the gravitational + electromagnetic geodesics classical equation):

$$
\left(V_{k} V^{k}\right)^{\frac{1}{2}}+\frac{e}{m c^{2}} A_{k} V^{k}=1
$$

At first sight, eq. 34 may seem not to be scale invariant. But one must not forget that $i \lambda \partial_{k}$ is a velocity operator, so that eq. 34 is actually homogeneous in the velocity variables.

\subsection{On the scale metric}

In Galilean scale-relativity, the invariant is actually the fractal dimension itself, $D=2=$ cst. In special scale-relativity [12], the scale-invariant takes a Minkowskian form, and writes in two dimensions:

$$
d \sigma^{2}=d \delta^{2}-\frac{(d \ln \mathcal{L})^{2}}{C^{2}}
$$

where $C=\ln \left(\lambda / \Lambda_{p}\right), \Lambda_{p}$ being the Planck length and $\lambda$ the fractal / nonfractal transition scale. Under this form, the meaning of the invariant $\sigma$ is straighforward. In the same way as, in special motion-relativity, the invariant gives the proper time in rest frame, here the scale invariant can be interpreted as a "proper scale dimension". In other word, in a scale-covariant representation, the now variable scale dimension $\delta$ is only a projected coordinate of a 5-dimensional vector, so that a proper fractal dimension, that keeps the value $D=2$, can still be defined.

\section{CONCLUSION}

By way of conclusion, let us recall how, in scale-relativity, the classical 4dimensional space-time can "emerge" from a 2-dimensional space [1] [2]. We have found, from special scale-relativity, that the fractal dimension tends to infinity when the resolution interval tends to zero (more precisely, in our framework, to the Planck length-scale). Such a conclusion is also reached by El Naschie [30] in his analysis of the set of the global fractal dimensions of spacetime. It is therefore possible to define a fractal curvilinear coordinate system in terms of which all the events of space-time could be reached by a unique fractal 
coordinate. A second coordinate is necessary for the description, namely, the scale dimension or "zoom" considered as variable.

Now, when going back to large space-time scales: (i) jumping to curvilinear non fractal coordinates, one recovers a 5 -dimensional space-time-zoom; (ii) at larger scale, the scale-dimension jumps to a constant value, then $d \delta=0$ and the 5 -space degenerates into a 4 -space; (iii) among the 4 variables $X^{k}$, we can singularize the one which is characterized by the smallest fractal-nonfractal transition, say $X^{1}$, and identify it with the time coordinate; then one can demonstrate ([1], pp.123-4) that the fractal divergences cancel in such a way that the three quantities $<d X^{k} / d X^{1}>, i=2,3,4$, remain smaller than 1 (i.e., smaller than $c$ in units $\mathrm{m} / \mathrm{s}$ ); (iv) this implies Einstein's motion-relativity, i.e., equivalently, a Minkowskian metric for the mean (classical) remaining 4 variables. Let us conclude by remarking that, at very high energy, when approaching the Planck mass scale, the physical description is reduced to that of the "quantum" variables $\xi$ 's, while the average, classical variables $x$ 's lose their physical meaning: we recover by another way the fact that gravitation and quantum effects become of the same order, and that a theory of quantum gravity is needed. However, the variables with which such a theory must be constructed are the $\xi$ 's rather than the $x$ 's. The fact that they are positive-definite instead of being of signature $(+,-,-,-)$ justifies the need to work in the framework of an Euclidean rather than Minkowskian space-time when performing path integral calculations in quantum gravity. However, if one wants to include our new interpretation of the Planck length-scale and to build a special scale relativistic theory, one must jump again to a hyperbolic signature in 5 dimensions.

It is a pleasure to acknowledge very interesting discussions with Drs. M. El Naschie, G. Ord and C. Castro.

\section{References}

[1] Nottale, L., 1993, Fractal Space-Time and Microphysics: Towards a Theory of Scale Relativity (World Scientific)

[2] Nottale, L., 1996a, Chaos, Solitons \& Fractals, 7, 877

[3] Ord, G.N., 1983, J. Phys. A: Math. Gen., 16, 1869

[4] Nottale, L., 1989, Int. J. Mod. Phys. A4, 5047

[5] Einstein, A., 1916, Annalen der Physik 49, 769.

[6] El Naschie, M., in Quantum Mechanics, Diffusion and Chaotic Fractals, Eds. M.S. El Naschie, O.E. Rössler and I. Prigogine (Pergamon, 1995).

[7] Damour, T., in 300 years of Gravitation, Eds. S.W. Hawking and W. Israel, Cambridge University Press, 1987, p. 128. 
[8] Castro, C., 1997, Found. Phys. Lett. 10, 273

[9] Nottale, L., in "Relativity in General", E.R.E. 93 (Spanish Relativity Meeting), Ed. J. Diaz Alonso and M. Lorente Paramo, pp.121-132, Editions Frontires (1994).

[10] Nottale, L., in Quantum Mechanics, Diffusion and Chaotic Fractals, Eds. M.S. El Naschie, O.E. Rössler and I. Prigogine (Pergamon, 1995), p. 51.

[11] Hermann, R., 1997, J. Phys. A 30, 3967

[12] Nottale L., 1992, Int. J. Mod. Phys. A7, 4899

[13] Nottale, L., 1997, in "Scale invariance and beyond", proceedings of Les Houches school, Ed. B. Dubrulle, F. Graner and D. Sornette, (EDP Sciences / Springer), p. 249

[14] Nottale, L., 1997, Astron. Astrophys., 327, 867

[15] Nottale, L., in Ciel et Terre, Bulletin de la Société Royale Belge d'Astronomie, in press

[16] Nottale, L., Schumacher, G., \& Gay, J., 1997, Astron. Astrophys , 322, 1018

[17] Nottale, L. 1996, Astron. Astrophys. Lett, 315, L9

[18] Nottale, L. 1998, Chaos, Solitons \& Fractals, 9, 1043

[19] Nottale, L. 1998, Chaos, Solitons \& Fractals, 9, 1035

[20] Hermann, R., Schumacher, G., Guyard, R., 1998, Astron. Astrophys. 335, 281.

[21] Hermann, R., in Proceedings of NATO Conference, "The dynamics of small bodies in the solar system", 1998, Dordrecht, in press

[22] Nottale, L., Lefèvre, E.T., \& Schumacher, G., 1997, in preparation

[23] Nottale, L., Tricottet, M., 1998, in preparation

[24] Pissondes, J.C., 1998, Chaos, Solitons \& Fractals 9, 1115

[25] Nottale, L., and Schneider, J., 1984, J. Math. Phys. 25, 1296

[26] Ord, G., 1996, Annals of Physics 250, 63

[27] Finkel, R.W., 1987, Phys. Rev. A 35, 1486

[28] Abbott, L.F., \& Wise, M.B., 1981, Am. J. Phys. 49, 37.

[29] Kröger, H., 1997, Phys. Rev. A 55, 951

[30] El Naschie, M., 1998, Chaos, Solitons \& Fractals 9, 931 